\title{
Embelin prevents LMP1-induced TRAIL resistance via inhibition of XIAP in nasopharyngeal carcinoma cells
}

\author{
SHU YANG, SHI-SHENG LI, XIN-MING YANG, DAN-HUI YIN and LIN WANG \\ Department of Otolaryngology, Head and Neck Surgery, The Second Xiangya Hospital, \\ Central South University, Changsha, Hunan 410011, P.R. China
}

Received February 22, 2015; Accepted March 15, 2016

DOI: $10.3892 / 01.2016 .4522$

\begin{abstract}
The tumor necrosis factor-related apoptosis-inducing ligand (TRAIL) selectively induces apoptosis in the majority of tumor cells, whilst sparing normal cells. However, the potential use of TRAIL in the treatment of cancer is limited by the inevitable emergence of drug resistance. The present study reports the upregulation of latent membrane protein 1 (LMP1)-induced TRAIL resistance via the enhanced expression of X-linked inhibitor of apoptosis protein (XIAP) in nasopharyngeal carcinoma (NPC) cells. LMP1-positive NPC cells were indicated to be more sensitive to TRAIL compared with LMP1-negative NPC cells in three NPC cell lines. CNE-1 is a LMP1-negative NPC cell line that was transfected with pGL6-LMP1; following which, sensitivity to TRAIL decreased. LMP1-induced TRAIL resistance was associated with the decreased cleavage of caspase- $8,-3$ and -9 , BH3 interacting domain death agonist (Bid) and mitochondrial depolarization, without any effects on the expression of the death receptors, B-cell lymphoma (Bcl)-2 and Bcl-extra long. Knockdown of XIAP with small interfering RNA increased caspase- 3 and -9 and Bid cleavage, and prevented LMP1-induced TRAIL resistance. Furthermore, embelin, the inhibitor of XIAP, prevented LMP1-induced TRAIL resistance in the Epstein-Barr virus (EBV)-positive CNE-1-LMP1 and C666-1 NPC cell lines. However, embelin did not enhance TRAIL-induced apoptosis in NP-69, which was used as a benign nasopharyngeal epithelial cell line. These data show that LMP1 inhibits TRAIL-mediated apoptosis by upregulation of XIAP. Embelin may be used in an efficacious and safe manner to prevent LMP1-induced TRAIL resistance. The present study may have implications for the development and
\end{abstract}

Correspondence to: Dr Shi-Sheng Li, Department of Otolaryngology, Head and Neck Surgery, The Second Xiangya Hospital, Central South University, 139 Renmin Road, Changsha, Hunan 410011, P.R. China

E-mail: lissdoctor@sina.com

Key words: nasopharyngeal carcinoma, latent membrane protein 1, tumor necrosis factor-related apoptosis-inducing ligand, apoptosis, X-linked inhibitor of apoptosis protein, embelin validation of novel strategies to prevent TRAIL resistance in EBV-positive NPC.

\section{Introduction}

Tumor necrosis factor (TNF)-related apoptosis inducing ligand (TRAIL) is a cytokine that belongs to the TNF family of proteins, and is able to induce apoptosis in a variety of cancer cells without significantly affecting normal cells (1). TRAIL promotes apoptosis by binding to certain cognate receptors, including TRAIL-R1, or death receptor 4 (DR4), and TRAIL-R2, or death receptor 5 (DR5) (2). Fas-associated death domain (FADD) and caspase-8 are aggregated and recruited to the DR-TRAIL complex to form the death-inducing signaling complex (DISC) (2). This apoptotic signal is additionally transmitted via extrinsic and intrinsic pathways (3). The extrinsic pathway is activated in a mitochondrial-independent mechanism. Upon recruitment, caspase- 8 is activated and results in the direct cleavage of downstream effector caspases such as caspase-3 to induce apoptosis (3). The intrinsic pathway is activated in a mitochondrial-dependent manner. Once cleaved by caspase-8, BH3 interacting domain death agonist (Bid) translocates to the mitochondria and activates the B-cell lymphoma (Bcl)-2 family members Bcl-2-associated $\mathrm{X}$ protein (Bax) and Bcl-2 killer 1 (Bak), which depolarize mitochondria (4). Apoptogenic factors, including cytochrome c, are released from the mitochondria into the cytosol to trigger the activation of caspase-3 to induce apoptosis (4). Studies have shown that certain cancer cells resist the apoptotic effect of TRAIL, an occurrence that is termed 'TRAIL resistance' (5). However, TRAIL resistance has been previously overcome by combining TRAIL with various chemotherapeutic drugs (6), which suggests that utilizing an approach that affects multiple targets may prevent or reverse TRAIL resistance in cancer cells. The development of effective therapies aimed at reversing TRAIL resistance is essential to effectively kill cancer cells.

Nasopharyngeal carcinoma (NPC) is common in Southeast Asia and in Southern parts of China $(3-5 / 10,000)$, but is rarely presented in other parts of the world (7). Until 1990, radiotherapy was the standard treatment for all stages of NPC (8). Despite significant progress in intensity-modulated radiation therapy and other types of therapy such as surgery and molecular targeted therapy, the outcomes for the patients remain disappointing, with five-year survival rates of $34-52 \%$ (9). 
Consequently, the identification of more effective treatments for NPC is required.

Death receptors have previously been detected in the biopsy specimens of head and neck tumors, but not in the surrounding normal tissues (10). This finding supports the hypothesis that the presence of death receptors in certain head and neck cancers may endow them with a greater susceptibility to TRAIL-mediated apoptosis. One study demonstrated the robust induction of apoptosis in the TW02 NPC cell line by TRAIL (11); however, another study indicated that inducing apoptosis in certain NPC cells lines by TRAIL may be challenging (12). The explanation for this discrepancy remains unknown. However, previous studies indicated that the overexpression of Bcl-2 and phosphatidylinositol-4,5-bisphosphate 3-kinase/protein kinase B activation may contribute to TRAIL resistance in NPC cell lines (13).

Persistent Epstein-Barr virus (EBV) infection may cause NPC, Hodgkin's lymphoma and Burkitt's lymphoma (14). Latent membrane protein 1 (LMP1) is the principle oncogene of EBV (15), and LMP1 immortalizes and transforms cells by controlling the signaling pathways that block apoptosis and stimulate cell proliferation (16). LMP1 induces chemoresistance and promotes cell survival in NPC $(17,18)$. Friboulet et al (19) showed that EBV-positive NPC cell lines expressed increased levels of inhibitor of apoptosis proteins (IAPs), which have anti-apoptotic functions. Additionally, X-linked inhibitor of apoptosis protein (XIAP) is a member of the IAP family that inhibits caspases and induces TRAIL resistance (20).

The present study aims to test the hypothesis that LMP1 overexpression induces TRAIL resistance in NPC cells by enhancing XIAP, and also aims to study the associated molecular mechanisms.

\section{Materials and methods}

Cell lines and reagents. Three human NPC cell lines, CNE-1, CNE-2 and C666-1, were used in the present study. CNE-1 is a well-differentiated squamous cell carcinoma NPC cell line that consistently expresses EBV, and was established by the Cancer Research Institute, Sun Yat-sen University (Guangdong, China). CNE-2 is poorly differentiated cell line that was derived from the primary tumor of a patient with poorly differentiated squamous cell carcinoma NPC and is positive for plasma EBV DNA (established by the Chinese Academy of Medical Sciences, Beijing, China). C666-1 is an NPC cell line that was established by the Department of Anatomical and Cellular Pathology, Prince of Wales Hospital, The Chinese University of Hong Kong (Shatin, Hong Kong, China).

The non-transformed nasopharyngeal epithelium NP-69 cell line was derived from the human nasopharynx, and established by the Department of Anatomy, Li Ka Shing Faculty of Medicine, University of Hong Kong (Hong Kong, China). All cells were cultured in RPMI-1640 media (Invitrogen; Thermo Fisher Scientific, Inc., Waltham, MA, USA) supplemented with $10 \%$ fetal bovine serum (FBS; Hyclone; GE Healthcare Life Sciences, Logan, UT, USA) and 100 U/ml penicillin/streptomycin (Huabei Pharmacy Group Xiantai Medicine Co., Ltd., Shijiazhuang, China). Cultures were maintained in a fully-humidified atmosphere of $5 \% \mathrm{CO}_{2}$ in air at $37^{\circ} \mathrm{C}$. TRAIL was obtained from Pfizer, Inc. (New York, NY,
USA). Embelin was obtained from Enzo Life Sciences, Inc. (Farmingdale, NY, USA). Embelin was dissolved in dimethyl sulfoxide (DMSO) (Beyotime Institute of Biotechnology, Haimen, China) at a $500 \mu \mathrm{M}$ concentration, and stored at $-20^{\circ} \mathrm{C}$ until required. The following antibodies were obtained from the indicated sources: Mouse anti-LMP1 monoclonal antibody (Dako, Glostrup, Denmark), rabbit anti-caspase-9 polyclonal antibody, rabbit anti-caspase- 8 polyclonal antibody and rabbit anti-caspase-3 polyclonal antibody (Cell Signaling Technology, Danvers, MA, USA). Mouse anti-Bcl-2 polyclonal antibody, mouse anti-Bcl-extra long (Bcl-XL) polyclonal antibody, mouse anti-Bid antibody, and mouse anti-XIAP polyclonal antibody were obtained from Santa Cruz Biotechnology, Inc., (Dallas, TX, USA). All antibodies were used at a dilution of $1: 1,000$ or $1: 800$.

3-(4,5-dimethylthiazolyl)-2,5-diphenyl tetrazolium bromide (MTT) cell viability assay. Cell viability was determined using an MTT assay. NPC cells (5,000 cells/well) were plated in 96-well plates (Beyotime Institute of Biotechnology). Subsequent to adding the indicated TRAIL treatment doses $(0,20,40,60,80$ and $100 \mathrm{ng} / \mathrm{ml})$ for various amounts of time $(4,8,12,16,20$ and $24 \mathrm{~h})$, cells were incubated for $2 \mathrm{~h}$ with $0.5 \mathrm{mg} / \mathrm{ml}$ of MTT (Sigma-Aldrich, St. Louis, MO, USA), and DMSO was used to solubilize the formazan product. Control wells were treated with DMSO only. The optical density (OD) of each well was measured at $570 \mathrm{~nm}$ with a microplate reader (MA68II; X-Rite, Inc., Grand Rapids, MI, USA) (survival rate $\left.=\mathrm{OD}_{\text {treat }} / \mathrm{OD}_{\text {control }}\right)$.

Determination of apoptosis by annexin V/propidium iodide (PI) staining. Levels of TRAIL-mediated apoptosis were determined using the Annexin V/PI staining kit (catalogue no. P0018A; Beyotime Institute of Biotechnology). Subsequent to incubation with the indicated TRAIL treatment doses $(20,40,60,80$ and $100 \mathrm{ng} / \mathrm{ml})$ for various lengths of time $(4,8,12,18$ and $24 \mathrm{~h})$, cells were harvested, and the cell pellets were suspended in $500 \mu \mathrm{l}$ binding buffer $[10 \mathrm{mM}$ 4-(2-hydroxyethyl)-1-piperazineethanesulfonic acid ( $\mathrm{pH} 7.4$ ), $140 \mathrm{mM} \mathrm{NaCl}, 1 \mathrm{mM} \mathrm{MgCl}$, $5 \mathrm{mM} \mathrm{KCl}$ and $2.5 \mathrm{mM} \mathrm{CaCl}_{2}$ ] at a density of $1 \times 10^{6}$ cells $/ \mathrm{ml}$. Samples were incubated with $1 \mu \mathrm{l}$ annexin V-fluorescein isothiocyanate (FITC) and $5 \mu \mathrm{lPI}$ for $5 \mathrm{~min}$ at room temperature in the dark and measured on a FACSort flow cytometer (BD Biosciences, Franklin Lakes, NJ, USA). Annexin V-FITC and PI fluorescence were detected in the FL-1 (green) and FL-2 (red) channels, respectively. Data were analyzed using CellQuest Pro software version 2.7 (BD Biosciences).

LMP1 complementary DNA (cDNA) transfection. The LMP1-expression vector (pGL6-LMP1) and pGL6 vector were obtained from Beyotime Institute of Biotechnology. Transfection was performed using the Lipofectamine 2000 (Invitrogen; Thermo Fisher Scientific, Inc.), according to the manufacturer's protocol. In brief, cells were seeded in a 6-well plate (Beyotime Institute of Biotechnology) at a density of $10^{5}$ cells/well and cultured for $24 \mathrm{~h}$ until cells attained a confluence of $60-70 \%$. The vector pGL6-LMP1 or the negative pGL6 vector $(10 \mu \mathrm{g})$ was diluted in $100 \mu \mathrm{l}$ of Opti-MEM (Thermo Fisher Scientific, Inc.) for $5 \mathrm{~min}$ at room temperature. 
During the incubation period, $5 \mu$ l of Lipofectamine 2000 was diluted in $100 \mu \mathrm{l}$ Opti-MEM. These two mixtures were combined, mixed gently, incubated for $20 \mathrm{~min}$ at room temperature, and then incubated with the cells for $20 \mathrm{~min}$ at room temperature. Stably transfected clones were selected in medium containing $0.5 \mathrm{mg} / \mathrm{ml}$ geneticin sulfate (G418; Amresco Inc., Framingham, MA, USA). Established clones were grown in RPMI-1640 medium supplemented with $10 \%$ FBS containing $0.5 \mathrm{mg} / \mathrm{ml} \mathrm{G} 418$ sulfate (Beyotime Institute of Biotechnology).

Flow cytometric analysis of TRAIL receptors. NPC cells at a density of $1 \times 10^{6}$ cells were suspended in $500 \mu \mathrm{l}$ of the medium and incubated with $5 \mu \mathrm{l}$ of anti-DR4 (catalogue no. SAB4700541) or anti-DR5 (catalogue no. SAB1303637) polyclonal rabbit antibodies (dilution, 1:100; Sigma-Aldrich) for $1 \mathrm{~h}$. Subsequent to washing in phosphate-buffered saline (PBS) (Beyotime Institute of Biotechnology) three times (5 min each), FITC-conjugated goat anti-rabbit polyclonal antibody (dilution, 1:200; catalogue no. A0528; Beyotime Institute of Biotechnology) was added to the cell suspensions and incubated for $1 \mathrm{~h}$ on ice. Subsequent to rinsing in PBS, samples were analyzed with a FACSort flow cytometer. Data were analyzed using CellQuest Pro software version 2.7.

$X I A P$-small interfering RNA (siRNA) transfection. The siRNA-targeting XIAP messenger RNA (mRNA) was predesigned and validated according to the following sequences: Sense 5'-GGAGAUACCGUGCGGUGCUdTdT-3' and antisense 5'-AGCACCGCACGGUAUCUCCdTdT-3'. The negative control siRNA was designed by the Invitrogen brand (Thermo Fisher Scientific, Inc.) and had the following sequences: Sense 5'-UUCUCCGAACGUGUCACGUdTdT-3' and antisense 5'-ACGUGACACGUUCGGAGAAdTdT-3'. Cells were seeded into a 6 -well plate at a density of $10^{5}$ cells/well and cultured to a confluence of $60-70 \%$. Cells were then transfected with $100 \mathrm{nM}$ specific or nonspecific siRNA. The transfection was accomplished with $30 \mathrm{ml}$ INTERFERin (Polyplus transfection; Ozyme, St. Quentin-en-Yvelines, France). The siRNA transfection reagent was added to the siRNA duplex in $500 \mu \mathrm{l}$ of Opti-MEM. Subsequent to $48 \mathrm{~h}$ incubation, the transfected cells were treated with TRAIL.

Western blot analysis. Cells were washed three times for $5 \mathrm{~min}$ in PBS, and then dissolved in lysis buffer $(20 \mathrm{mM}$ $\mathrm{Na}_{2} \mathrm{PO}_{4}, 150 \mathrm{mM} \mathrm{NaCl}, 1 \%$ Triton $\mathrm{X}-100,1 \%$ aprotinin, $1 \mathrm{mM}$ phenylmethylsulfonyl fluoride, $100 \mathrm{mM} \mathrm{NaF}$ and $2 \mathrm{mM} \mathrm{Na}_{3} \mathrm{VO}_{4}$ ). Proteins were quantified with a Bradford kit (catalaogue no. P0006; Beyotime Institute of Biotechnology). Next, proteins (20 $\mu \mathrm{g} / \mathrm{lane})$ were separated by sodium dodecyl sulfate-polyacrylamide gel electrophoresis and transferred to a polyvinylidene difluoride membrane. Membranes were incubated with the primary antibody [mouse anti-LMP1 monoclonal antibody (catalogue nos. M0897, IR753 and IS753; Dako), rabbit anti-caspase-9 polyclonal antibody (catalogue no. 9506; Cell Signaling Technology), rabbit anti-caspase-8 polyclonal antibody (catalogue no. 9496s; Cell Signaling Technology), rabbit anti-caspase-3 polyclonal antibody (catalogue no. 9665s; Cell Signaling Technology), mouse anti-Bcl-2 polyclonal antibody (catalogue no. sc-7382; Santa Cruz Biotechnology, Inc.), mouse
anti-Bcl-XL polyclonal antibody (catalogue no. sc-8392; Santa Cruz Biotechnology, Inc.), mouse anti-Bid antibody (catalogue no. 5C9; Santa Cruz Biotechnology, Inc.) and mouse antiXIAP polyclonal antibody (catalogue no. sc-55552; Santa Cruz Biotechnology, Inc.)] overnight at $4^{\circ} \mathrm{C}$. The membranes were then washed in PBS three times for $5 \mathrm{~min}$, and incubated with horseradish peroxidase-conjugated goat anti-rabbit secondary antibodies (1:500; catalogue no. A0192; Beyotime Institute of Biotechnology). Proteins were detected using an enhanced chemiluminescence kit (catalogue no. P0018A; Beyotime Institute of Biotechnology).

Semi-quantitative reverse transcription-polymerase chain reaction $(R T-P C R)$. Total RNA was extracted and purified with TRIzol Total RNA Extraction Kit (catalogue no. R0016; Beyotime Institute of Biotechnology), using DNase (catalogue no. R0021; Beyotime Institute of Biotechnology). Total RNA $(1 \mu \mathrm{g})$ was reverse transcribed by High-Capacity cDNA Reverse Transcription Kits (Applied Biosystems; Thermo Fisher Scientific, Inc.), according to the manufacturer's protocol. The primers for LMP1 and $\beta$-actin were synthesized by Sangon Biological Engineering Technology and Services Co. Ltd. (Shanghai, China) as follows: LMP1, sense 5'-TGG AGGGAGAGTCAGTCAGGC-3' and antisense 5'-ATTGAC GGAAGAGGTTGAAAAC-3' to generate a 254-bp fragment; glyceraldehyde 3-phosphate dehydrogenase (GAPDH), sense 5'-ACCCACTCCTCCACCTTTG-3' and antisense 5'-ACC CACTCCTCCACCTTTG-3' to generate a 188-bp fragment. The RT products $(1 \mu \mathrm{l})$ were amplified by PCR using the following conditions: $95^{\circ} \mathrm{C}$ for $5 \mathrm{~min}$; then 32 cycles at $95^{\circ} \mathrm{C}$ for $30 \mathrm{sec}, 58^{\circ} \mathrm{C}$ for $30 \mathrm{sec}$ and $72^{\circ} \mathrm{C}$ for $30 \mathrm{sec}$; and a final extension at $72^{\circ} \mathrm{C}$ for $5 \mathrm{~min}$. PCR products were electrophoresed on $1.5 \%$ agarose gels at $100 \mathrm{~V}$ for $40 \mathrm{~min}$, using DNA ladder (catalogue no. D0107; Beyotime Institute of Biotechnology). Bands were visualized with ethidium bromide (Shanghai Rong Bo Biotechnology Co., Ltd., Shanghai, China). LMP1 mRNA expression levels were quantified by Quantity One software version 4.31 (Bio-Rad Laboratories, Inc., Hercules, CA, USA) and represented as the densitometric ratio of the targeted gene relative to GAPDH. PCR experiments were repeated three times.

Assessment of mitochondrial transmembrane potential $(\Delta \psi m)$. Assessment of the $\Delta \psi \mathrm{m}$ was performed using cationic lipophilic fluorochrome 3,3'-dihexyloxacarbocyanine iodide $\left[\mathrm{DiOC}_{6}(3) ; 460 \mathrm{ng} / \mathrm{ml}\right.$; Molecular Probes; Thermo Fisher Scientific, Inc.). The cells were treated for $12 \mathrm{~h}$ with $100 \mathrm{ng} / \mathrm{ml}$ TRAIL and incubated for $30 \mathrm{~min}$ at $37.8^{\circ} \mathrm{C}$ in complete medium consisting of DMSO with $\mathrm{DiOC}_{6}(3)$. The cells were then washed twice in PBS three times for $5 \mathrm{~min}$, and analyzed using a FACSort flow cytometer and analyzed with Cell Quest Pro software.

Statistical analysis. All tests were performed in triplicate and statistical analyses were performed using SPSS software (version 15.0; SPSS, Inc., Chicago, IL, USA). Descriptive statistics were expressed as the mean \pm standard deviation, and Student's $t$-tests were used to analyze the significant differences between paired datasets. An $\alpha$ value of $\mathrm{P}<0.05$ was considered to indicate a statistically significant difference. 
A

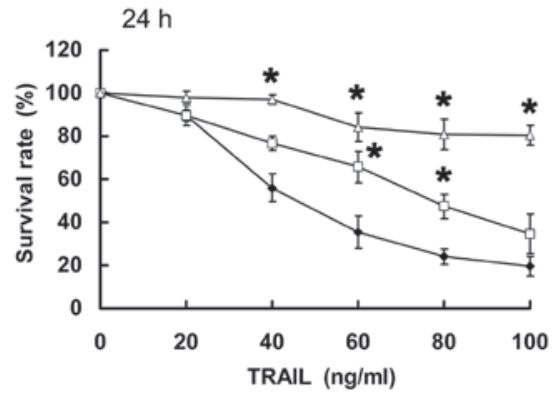

B

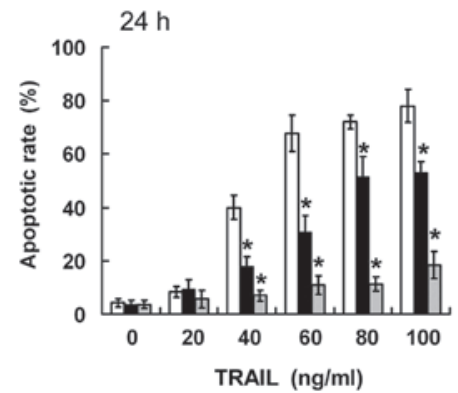

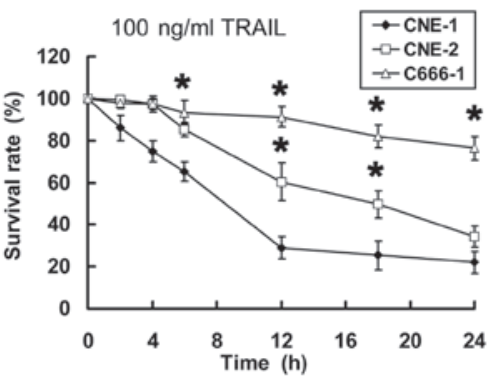

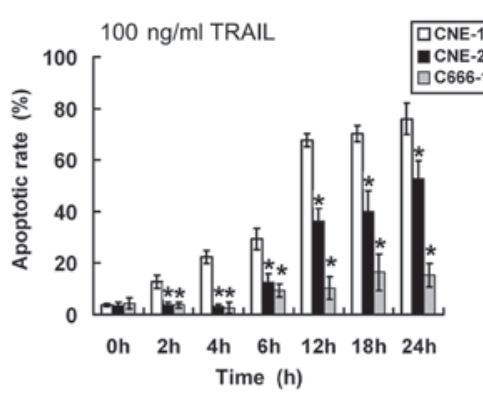

C

$\beta$-actin

A CNE-1 $\begin{gathered}\text { CNE-1 } \\ \text {-DGLE }\end{gathered}$-LMP1

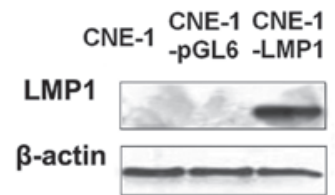

B LMP1

GAPDH $-\infty$

Figure 1. Expression of LMP1 was associated with the TRAIL sensitivity of NPC cell lines. (A) CNE-1, CNE-2 and C666-1 cells were treated with escalating

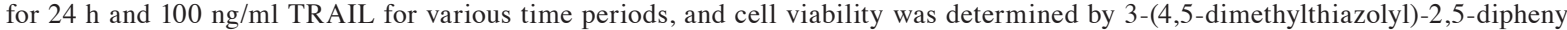
tetrazolium bromide assay. (B) Cells were treated with escalating doses of TRAIL for $24 \mathrm{~h}$ and $100 \mathrm{ng} / \mathrm{ml}$ TRAIL for various time periods, and apoptotic rate was determined by flow cytometric analysis. Apoptosis rate included early apoptosis (Annexin-V positive) and late apoptosis (propidium iodide positive) (C) Western blot analysis was used to detected LMP1 protein expression in three NPC cell-lines. "P $<0.05$ compared with CNE-1 cells. LMP1, latent membrane protein 1; TRAIL, tumor necrosis factor-related apoptosis-inducing ligand; NPC, nasopharyngeal carcinoma.
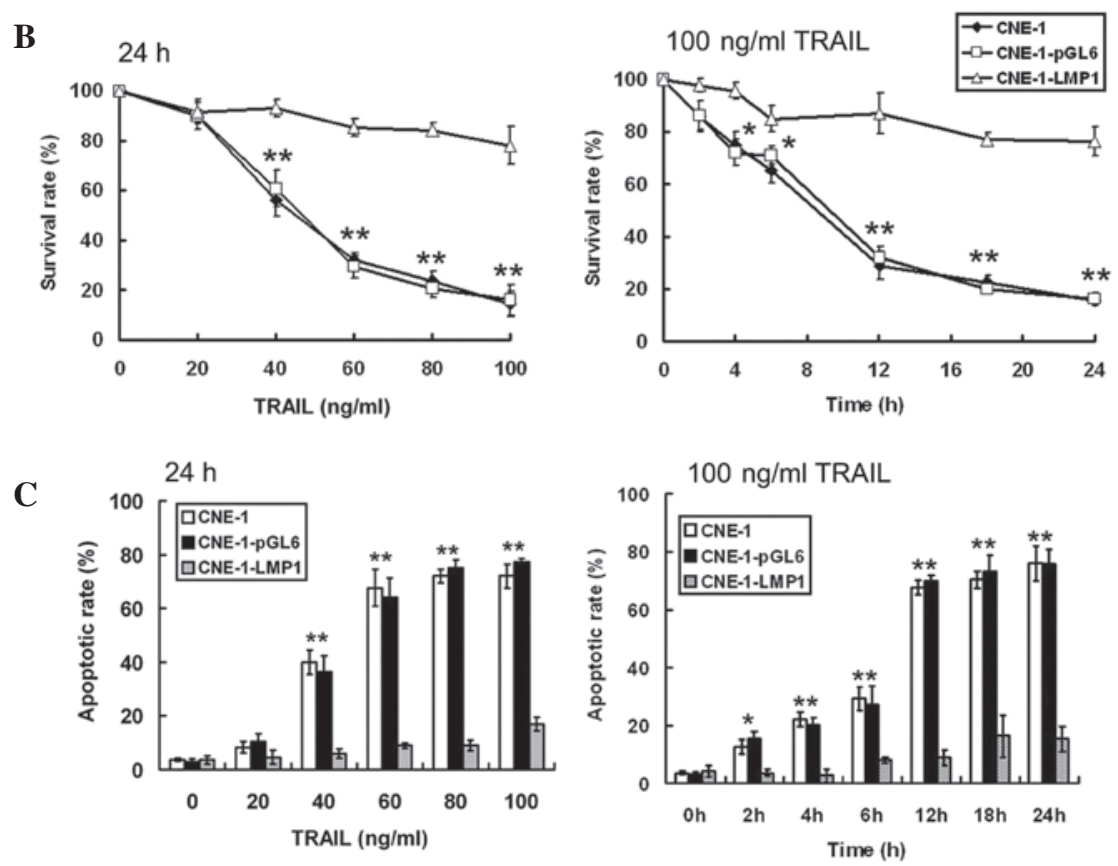

Figure 2. Overexpression of LMP1 protein induced TRAIL resistance in nasopharyngeal carcinoma cells. (A) Expression level of LMP1 was assessed by reverse transcription-polymerase chain reaction and western blot analysis, following transfection of pGL-6-LMP1 to CNE-1. (B) CNE-1, CNE-1-pGL6 and CNE-1-LMP1 were treated with escalating doses of TRAIL for $24 \mathrm{~h}$ or escalating times with $100 \mathrm{ng} / \mathrm{ml} \mathrm{TRAIL}$, respectively, and cell viability was determined by 3-(4,5-dimethylthiazolyl)-2,5-diphenyl tetrazolium bromide assay. (C) The cell apoptotic rate was determined by flow cytometry analysis. Apoptosis rate included early apoptosis (Annexin V positive) and late apoptosis (propidium iodide positive). ${ }^{*} \mathrm{P}<0.05$ compared with CNE-1-LMP1 cells; ${ }^{* * *} \mathrm{P}<0.01$ compared with CNE-1-LMP1 cells. LMP1, latent membrane protein 1; TRAIL, tumor necrosis factor-related apoptosis-inducing ligand; GAPDH, glyceraldehyde 3-phosphate dehydrogenase. 
A

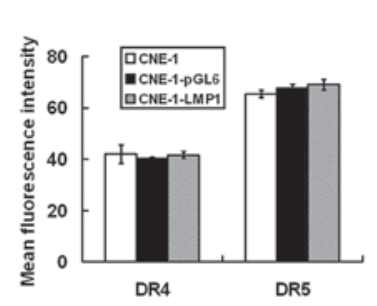

C
B

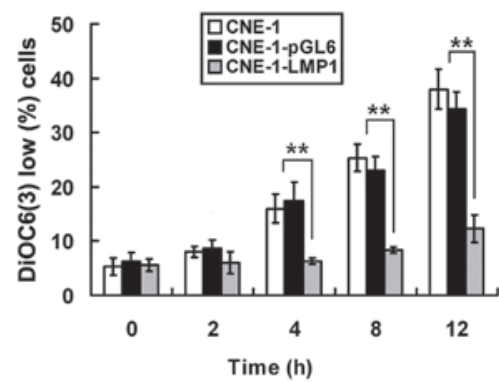

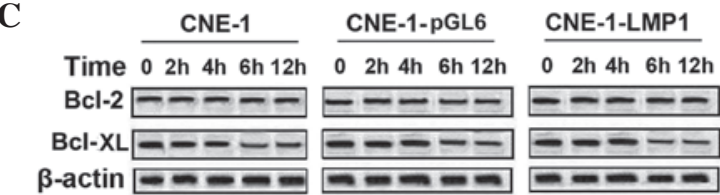

Figure 3. LMP1 induced TRAIL resistance via inhibition of mitochondrion-dependent apoptotic pathway. (A) Expressions of DR4 and DR5 were measured by flow cytometry analysis. (B) Mitochondrial depolarization was measured by $\mathrm{DiOC}_{6}(3)$ fluorescence during TRAIL treatment (100 ng/ml) for various periods of time. (C) Bcl-2 and Bcl-XL expression were assessed by western blot analysis during TRAIL treatment $(100 \mathrm{ng} / \mathrm{ml})$ for various periods of time. ${ }^{* *} \mathrm{P}<0.01$ compared with CNE-1-LMP1 cells. LMP1, latent membrane protein 1; TRAIL, tumor necrosis factor-related apoptosis-inducing ligand; DR4, death receptor 4; DR5, death receptor 5; Bcl-2, B-cell lymphoma-2; Bcl-XL, B-cell lymphoma-extra long; DiOC $_{6}(3), 3,3$ '-dihexyloxacarbocyanine iodide.

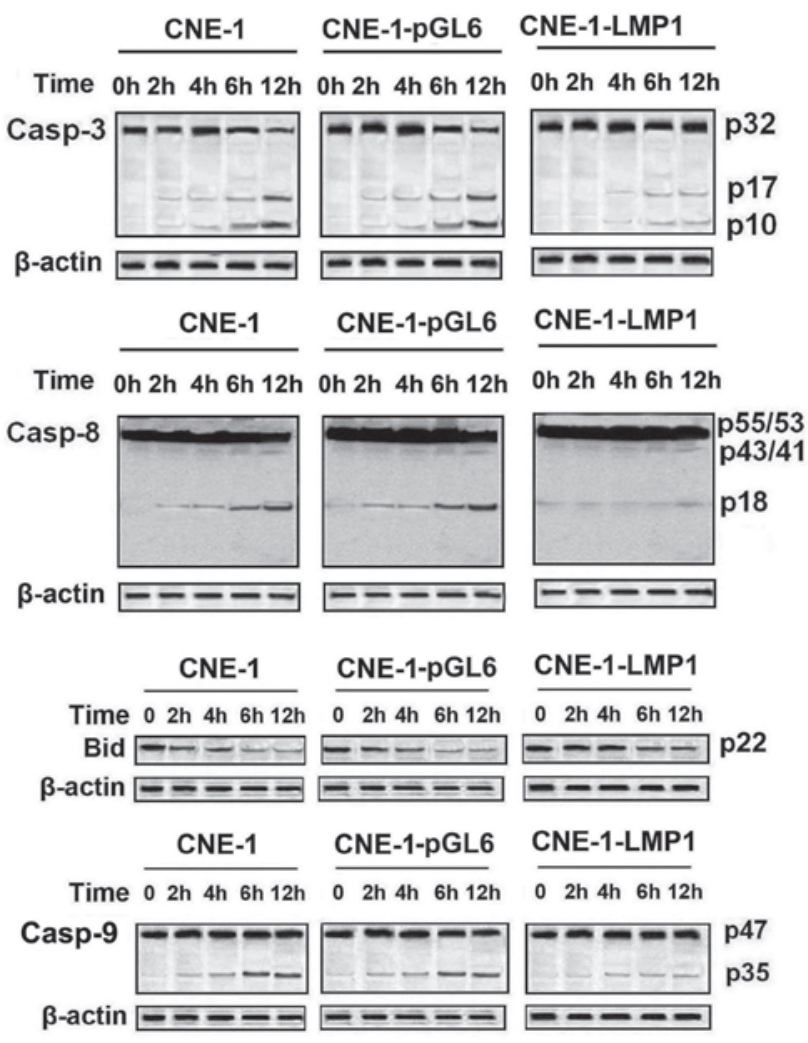

Figure 4. LMP1 induced TRAIL resistance via inhibition of caspase and Bid cleavage. Cleavage of caspases-3, -8 and -9 and Bid was assessed by western blot analysis during TRAIL treatment $(100 \mathrm{ng} / \mathrm{ml})$ for various periods of time. Caspase-3, p32-proform, p17, p10-cleavage fragments; caspase-8, p55/53-proform, p43/41, p18-cleavage fragments; caspase-9: p47-proform, p35-cleavage fragments. LMP1, latent membrane protein 1 TRAIL, tumor necrosis factor-related apoptosis-inducing ligand; $\mathrm{Bid}$, $\mathrm{BH} 3$ interacting domain death agonist.

\section{Results}

Sensitivity to TRAIL varies among NPC cells. First, the growth inhibition effects of TRAIL against NPC cell lines were assessed by MTT assay. TRAIL induced cell death in a doseand time-dependent manner. C666-1 cells were highly resistant to TRAIL, whereas CNE-1 cells were highly sensitive (Fig. 1A). The apoptosis rate was additionally analyzed by annexin V/PI staining and flow cytometry to confirm whether the difference in cell death was due to various apoptotic responses. The apoptotic effect of TRAIL was also time- and dose-dependent. The percentage of apoptotic cells was highest in CNE-1 cells and lowest in C666-1 cells in response to TRAIL treatment (Fig. 1B). LMP1 was detected in C666-1, but not in either CNE-1 or CNE-2 cells. The level of LMP1 expression was also tested in the non-transformed nasopharyngeal epithelial cell line NP-69, which did not show expression of LMP1 (Fig. 1C). From the results, LMP1-positive NPC cells were concluded to be more sensitive to TRAIL compared with LMP1-negative NPC cells.

Overexpression of LMPI inhibits the apoptotic effect of TRAIL. LMP1-positive NPC cells were indicated to be more sensitive to TRAIL compared with LMP1-negative NPC cells. Therefore, a genetic approach was used to upregulate LMP1 expression in CNE-1 cell lines. The pGL6-LMP1 vector was transfected into CNE-1 cells. Stable pGL6-LMP1 transfected cells and empty vector transfected control cells were designated as CNE-1-LMP1 and CNE-1-pGL6, respectively. Semi-quantitative RT-PCR and western blot analyses revealed that LMP1 expression was enhanced in CNE-1-LMP1 cells (Fig. 2A). The effect of TRAIL on cell survival was evaluated by MTT cell viability assays (Fig. 2B). Treatment with TRAIL resulted in cell death in CNE-1 and CNE-1-pGL6 cells in a dose- and time-dependent manner but had a dampened effect on CNE-1-LMP1 cells. Similarly, TRAIL induced significant apoptosis in CNE-1 and CNE-1-pGL6 cells in a time- and dose-dependent manner, but the apoptosis level was not as high in CNE-1-LMP1 cells (Fig. 2C). The results suggested that the overexpression of LMP1 may inhibit TRAIL-induced apoptosis.

Overexpression of LMP1 inhibits mitochondrial-dependent apoptosis in NPC cells. To elucidate the molecular mechanism 
A

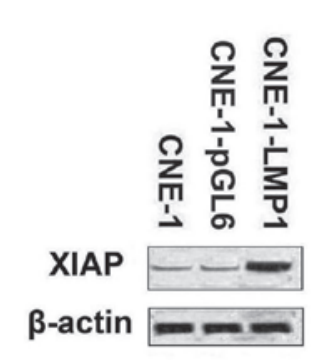

C

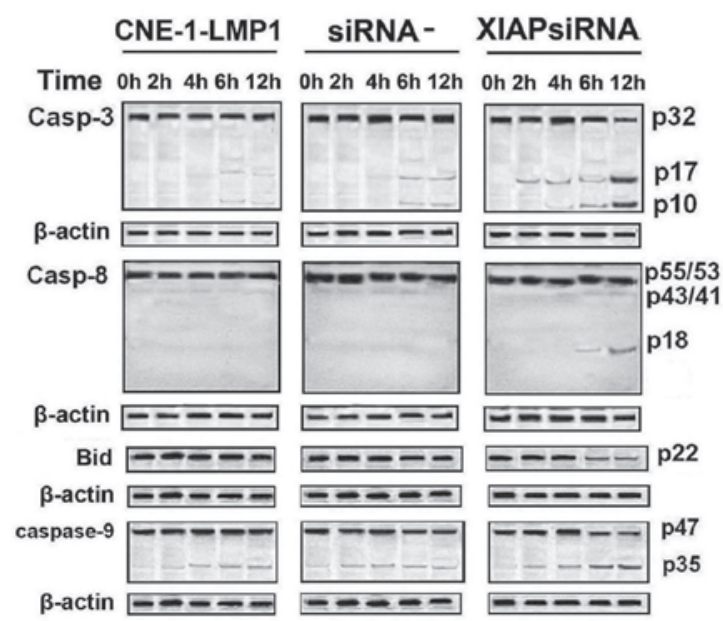

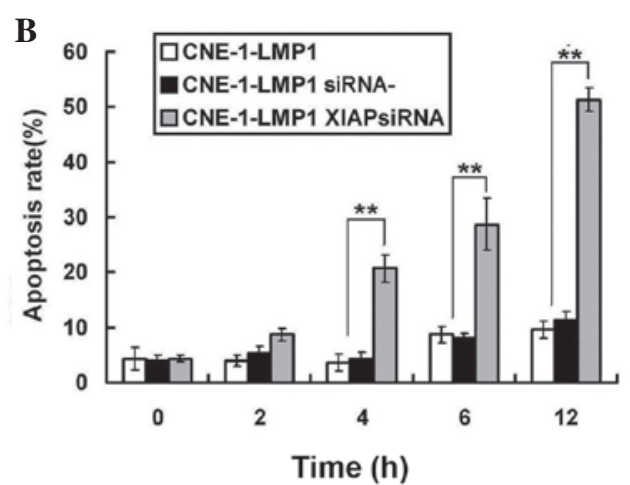

D

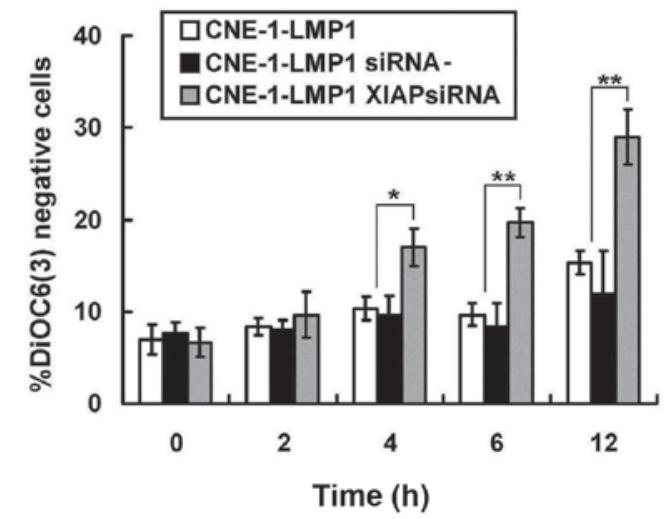

Figure 5. Upregulation of XIAP was involved in LMP1-induced TRAIL resistance. (A) Expressions of XIAP were assessed by western blot analysis. (B) Upon treatment with TRAIL (100 ng/ml) for various periods of time, apoptosis in CNE-1-LMP1 transfected with XIAP-siRNA was determined by flow cytometric analysis. (C) Upon treatment with TRAIL (100 ng/ml) for various periods of time, caspase-3, -8 and -9 and Bid cleavage was assessed by western blot analysis in CNE-1-LMP1 cells transfected with XIAP-siRNA. (D) Upon treatment with TRAIL (100 ng/ml) for various periods of time, mitochondrial depolarization was measured by $\mathrm{DiOC}_{6}(3)$ fluorescence in CNE-1-LMP1 cells transfected with XIAP-siRNA. siRNA-, CNE-1-LMP1 transfected with negative control siRNA; XIAP-siRNA, CNE-1-LMP1 transfected with XIAP-siRNA. "P<0.05 compared to CNE-1-LMP1 transfected with XIAP-siRNA; ** P<0.01 compared to CNE-1-LMP1 cells transfected with XIAP-siRNA. XIAP, X-linked inhibitor of apoptosis protein; LMP1, latent membrane protein 1 TRAIL, tumor necrosis factor-related apoptosis-inducing ligand; siRNA, small interfering RNA; Bid, BH3 interacting domain death agonist; DiOC 6 (3), 3,3'-dihexyloxacarbocyanine iodide.

responsible for LMP1-indued TRAIL resistance, the effect of LMP1 on the TRAIL-induced apoptotic pathway was examined. Since TRAIL is known to trigger apoptosis via binding to DR4 and DR5, the expression of DR4 and DR5 was examined (Fig. 3A). LMP1 overexpression did not change the cell surface expression levels of either DR4 or DR5 $(\mathrm{P}=0.087)$. Furthermore, the $\mathrm{DiOC}_{6}(3)$ negative cell rate was increased in CNE-1 and CNE-1-pGL6 cells compared with the CNE-1-LMP1 cells during TRAIL treatment. This result suggested that mitochondrial depolarization was inhibited by LMP1 overexpression (Fig. 3B). The expression levels of the pro-survival proteins Bcl-2 and Bcl-XL, were unaffected by LMP1 overexpression (Fig. 3C). The results showed that LMP1 inhibited the mitochondrial-dependent apoptotic pathway without having any impact on Bcl-2 and Bcl-XL.

LMP1 inhibits TRAIL-induced caspase and Bid cleavage in NPC cells. Analysis of caspase activation showed that the cleaved, mature forms of caspase-8 (p43/41), caspase-9 (p35) and caspase-3 (p17, p10) were generated in CNE-1 and CNE-1-pGL6 cells during TRAIL treatment, in a time-dependent manner (Fig. 4). However, CNE-1-LMP1 showed minimal caspase activation during TRAIL treatment. This observation indicated that LMP1 inhibited TRAL-induced caspase activation. Previously, the present study demonstrated that LMP1 inhibited TRAIL-induced mitochondrial-dependent apoptotic pathways. Therefore, the effects of LMP1 on TRAIL-induced Bid cleavage, which is an important event in mitochondrial-dependent apoptotic pathways, were additionally examined. Since the anti-Bid antibody recognized only the uncleaved Bid protein and not the cleaved product, the cleavage of Bid was assessed by determining the extent of the decreased levels of uncleaved Bid protein. TRAIL treatment resulted in a reduction in uncleaved Bid (indicating Bid cleavage) in CNE-1 and CNE-1-pGL6 cells. However, the reduction in Bid was inhibited in CNE-1-LMP1 cells, suggesting that LMP1 affected the upstream cleavage of Bid.

XIAP is involved in LMPl-induced TRAIL resistance in $N P C$ cells. Based on the initial results that showed that caspase activation was inhibited by LMP1 overexpression, the expression of XIAP proteins, which are known to antagonize caspase activity, were analyzed. Western blot analysis 
A

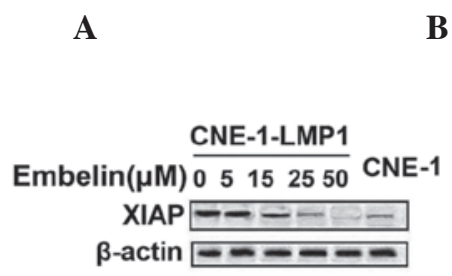

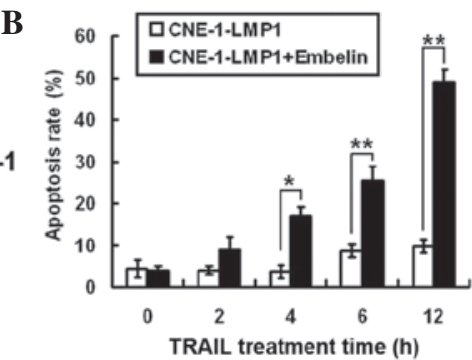

C

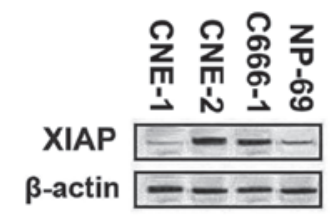

D

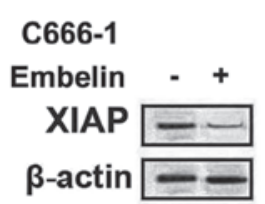

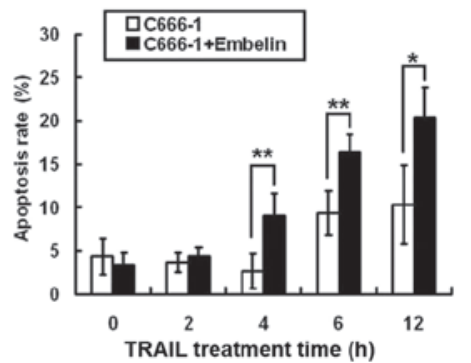

$\mathbf{E}$

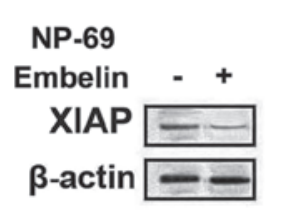

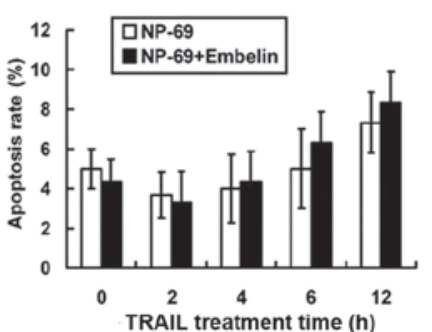

Figure 6. XIAP special inhibitor embelin inhibited XIAP expression and prevented LMP1-induced TRAIL resistance. (A) Upon treatment with various does of embelin for $24 \mathrm{~h}$, XIAP expression in CNE-1-LMP1 cells was assessed by western blot analysis. (B) Apoptotic rate was determined by flow cytometry analysis. (C) XIAP expression in four cell-lines (CNE-1, CNE-2, C666-1 and NP-69) was assessed by western blot analysis. (D) Upon treatment with $25 \mu \mathrm{M}$ embelin for $24 \mathrm{~h}$, XIAP expression in C666-1 cells was assessed by western blot analysis and apoptotic rate was determined by flow cytometric analysis following treatment with TRAIL. (E) Upon treatment with $25 \mu \mathrm{M}$ embelin for $24 \mathrm{~h}$, XIAP expression in NP-69 cells was assessed by western blot analysis and apoptotic rate was determined by flow cytometric analysis following treatment with TRAIL. "P $<0.05$ compared with cells treated with embelin; ${ }^{* *} \mathrm{P}<0.01$ compared to cells treated with embelin. XIAP, X-linked inhibitor of apoptosis protein; LMP1, latent membrane protein 1 TRAIL, tumor necrosis factor-related apoptosis-inducing ligand.

revealed that XIAP was upregulated in CNE-1-LMP1 cells (Fig. 5A). To determine the role of XIAP in LMP1 function, the expression of XIAP in CNE-1-LMP1 cells was knocked-down by siRNA (Fig. 5B). XIAP was indicated to be efficiently inhibited by the specific siRNA, and the apoptotic rate of CNE-1-LMP1 cells was increased during TRAIL treatment $(\mathrm{P}=0.021)$. Upon treatment with TRAIL, the extent of XIAP downregulation increased the cleavage of caspase-3, -8 and -9 and Bid in CNE-1-LMP1 cells (Fig. 5C). Furthermore, as shown in Fig. 5D, the decreased expression of XIAP increased the $\mathrm{DiOC}_{6}(3)$ negative cell rate. These observations suggest that XIAP was involved in LMP1-induced TRAIL resistance in NPC cells.

The XIAP special inhibitor, embelin, prevented LMPl-indued TRAIL resistance. As previously mentioned, XIAP was involved in LMP1-induced TRAIL resistance in NPC cells. Therefore, the present study sought to determine whether embelin (XIAP inhibitor) prevents LMP1-induced TRAIL resistance. CNE-1-LMP1 cells were treated with various concentrations of embelin $(5,15,25$ and $50 \mu \mathrm{M})$ for $24 \mathrm{~h}$ (Fig. 6A). The level of protein expression of XIAP in CNE-1-LMP1 cells was indicated to be decreased to the level observed in CNE-1 cells, following treatment with $25 \mu \mathrm{M}$ embelin. Additionally, embelin pretreated CNE-1-LMP1 cells, co-treated with TRAIL, revealed that embelin pretreatment increased TRAIL-induced apoptosis in CNE-1-LMP1 cells (Fig. 6B).

Embelin prevented the LMPI-indued TRAIL resistance. As embelin prevented LMP1-induced TRAIL resistance, the effect of embelin on C666-1 cells, the EBV-positive NPC cell line, was examined. The expression of XIAP was determined in three NPC cell lines and in NP-69 cells. Notably, XIAP expression levels were not increased in C666-1 cells. However, CNE-2 demonstrated the greatest expression levels of the cell lines (Fig. 6C). C666-1 cells were treated with $25 \mu \mathrm{M}$ of embelin for $24 \mathrm{~h}$ to dampen XIAP expression, following TRAIL treatment. The apoptotic effect of the combined treatment was stronger compared with treatment with TRAIL alone (Fig. 6D). The results show that embelin enhanced TRAIL sensitivity of EBV-positive NPC cells.

The interest in TRAIL is due in large part to its selective tumoricidal effects and relatively low toxicity profile towards normal cells. The present study selected NP-69, which was used as a model of the benign nasopharyngeal epithelium cell line to test the toxicity of combined treatment against benign cells (Fig. 6E). Treatment with embelin was indicated to effectively decrease XIAP protein levels and not to enhance TRAIL-induced apoptosis in NP-69 cells.

\section{Discussion}

The present study indicated that LMP1-positive NPC cells are more sensitive to TRAIL compared with LMP1-negative NPC cells. Therefore LMP1 was hypothesized to induce TRAIL resistance in NPC cells. CNE-1-LMP1 cell lines that express LMP1 were established, and the overexpression of LMP1 was indicated to induce TRAIL resistance. The knockdown of XIAP by siRNA showed that the upregulation of XIAP was involved in mediating the role of LMP1. In addition, the XIAP inhibitor, embelin, suppressed the expression of the XIAP protein and prevented LMP1-induced TRAIL resistance in CNE-1-LMP1 and EBV-positive C666-1 NPC cells. The present study showed that LMP1 induces TRAIL resistance in NPC cells via XIAP upregulation. Furthermore, embelin may 
be additionally evaluated as an agent for combination therapy with TRAIL in EBV-positive NPC patients.

At present, numerous factors have been identified that may affect TRAIL-induced apoptosis. However, the underlying molecular mechanisms remain to be defined. Snow et al (21) reported that resistance to TRAIL-induced apoptosis was observed in EBV-positive spontaneous lymphoblastoid cell lines that were derived from patients with post-transplant lymphoproliferative disease. This observation suggested a role for EBV in TRAIL resistance. The expression of EBV genes is indicated in the majority of NPC cell lines (22). These genes encode viral proteins that may contribute to malignant phenotypes (15). Among these genes, LMP1 is considered a primary viral oncogene (23). The presence of LMP1 in NPC tissues suggests the contribution of LMP1 to EBV-mediated tumorigenesis (24). Silencing of the LMP1 gene by siRNA enhances the chemosensitivity of EBV-positive NPC cells to bleomycin and cisplatin, suggesting a possible role for LMP1 in chemoresistance in NPC (17).

The present study tested the effect of LMP1 on TRAIL-induced apoptosis in NPC cells. C666-1 cells were indicated to be less sensitive to TRAIL compared with CNE-1 and CNE-2. A genetic approach was used to upregulate LMP1 in CNE-1, and the overexpression of LMP1 decreased sensitivity to TRAIL via the inhibition of mitochondrial depolarization. By contrast, Zhang et al (25) indicated that LMP1 increased cisplatin-induced mitochondrial depolarization and apoptosis in cervical carcinoma-derived HeLa cells. This inconsistency between findings may be due to the varying apoptotic mechanisms induced by cisplatin and TRAIL. Bcl-2 or Bcl-XL overexpression during Fas-induced apoptosis defines the two types of cells as having a differential dependence on the mitochondrial pathway. In type II cells, where apoptosis is associated with a mitochondrial-dependent pathway, the overexpression of $\mathrm{Bcl}-2$ or $\mathrm{Bcl}-\mathrm{XL}$ prevents TRAIL-induced apoptosis (26). The present study indicated that TRAIL induced mitochondrial depolarization in CNE-1 cells, such that $\mathrm{Bcl}-2$ and $\mathrm{Bcl}-\mathrm{XL}$ may potentially regulate the sensitivity of CNE-1 cells to TRAIL. However, Bcl-2 and Bcl-XL were unaffected by the increased expression of LMP1, suggesting that LMP1 regulates TRAIL-induced apoptosis without the effect on Bcl-2 and Bcl-XL. It has been previously reported that LMP1 augments Bcl-2 expression in certain cells, including B cells and certain types of NPC cell lines $(27,28)$. LMP1 may therefore differentially modulate Bcl-2 expression in various cell types and cell differentiated types.

The present study also indicated that the TRAIL-induced cleavage of caspase- $8,-3$ and -9 was inhibited by the enhanced expression of LMP1. 'Initiator' caspases with long prodomains, such as caspase-8, either directly or indirectly activate 'effector' caspases, such as caspase-3 (29). Vier et al (30) showed that the enhancement of death receptor expression induces caspase- 8 cleavage. However, the present study demonstrated that the death receptors were expressed at similar levels in CNE-1, CNE-1-pGL6 and CNE-1-LMP1 cells, suggesting that LMP1 inhibited caspase- 8 cleavage via an additional mechanism. For example, a previous study indicated that LMP1 inhibited caspase-8 cleavage through the upregulation of the FLIP protein, which possesses homology to caspase-8 (31). Following Bid cleavage, caspase- 9 is activated and mitochondria are disrupted to activate caspase-3. In the present study, these events were inhibited by LMP1 upregulation. Consistent with the results of the present study, Kijima et al (14) knocked-down the LMP1 gene in NPC cells and identified that the cleavage of caspase-3 and -8 and poly(ADP-ribose) polymerase 1 was increased. However, in another study, LMP1 activated caspase-8, -9, -3 and -7 via the induction of Fas overexpression in lymphoblastoid cell lines (32). The inconsistencies observed between these studies may be attributed to the dual effects of LMP1 to induce apoptosis, which was also observed in the study conducted by Chen and Chen (33).

In the present study, the enhanced expression of XIAP was indicated to be involved in LMP1-induced TRAIL resistance. IAPs are a group of anti-apoptotic factors that render cancer cells insensitive to apoptotic stimulation (34). A previous study considered XIAP to be the most potent IAP member for apoptosis inhibition and to often be overexpressed in various cancers (35). Furthermore, the overexpression of XIAP in cancer is often associated with a poor prognosis and resistance to chemotherapy $(36,37)$. Certain studies have clearly demonstrated that XIAP may inhibit TRAIL-induced apoptosis through the inactivation of caspase-3 and -9 (38). Consistent with previous reports, the present study indicated that the downregulation of XIAP reversed LMP1-induced TRAIL resistance by a mechanism that was dependent on increased mitochondrial depolarization and the cleavage of caspase-3 and -9 . XIAP inhibits apoptosis by binding to, and thereby inactivating, certain caspases, including the initiator caspase-9 and the effector caspases-3 and $-7(39,40)$. Previously, the binding affinity of XIAP to caspase- 3 and -7 was indicated to be increased compared with the binding affinity to the initiator caspase-9; whereas the binding affinity of XIAP to the initiator caspase- 8 was undetectable (35). This affinity profile places XIAP as an inhibitor of the common effector phase of apoptosis; therefore, XIAP acts downstream of the Fas death-receptor stimulation and the receptor proximal signaling events within the DISC, including FADD-mediated recruitment and activation of caspase-8. By contrast, and concordant with previous reports, the present study indicated that the downregulation of XIAP increased the cleavage of caspase-8 (35). Ferreira et al (41) indicated that in the hepatocytic type I Fas signaling pathway, active p17 (a claved fragment of caspase-3) feeds back to caspase-8, which cleaves the partially processed $\mathrm{p} 43$ fragment into the fully processed p18 species. XIAP siRNA-induced caspase- 8 activation may, therefore, be attributed to caspase-3 activation feedback. In addition, the downregulation of XIAP in the present study resulted in decreased Bid expression levels in response to TRAIL treatment. This observation suggested that enhanced LMP1-induced XIAP expression reduced sensitivity to TRAIL via the inhibition of Bid cleavage.

Embelin has previously been shown to have antitumor, anti-inflammatory and analgesic properties (42). Embelin has also been identified as a small molecular inhibitor of XIAP (43). Certain studies have shown that embelin suppresses the expression of XIAP and sensitizes tumor cells to TRAIL-induced apoptosis (44). The present study tested whether embelin could inhibit the LMP1-mediated upregulation of XIAP and TRAIL resistance. XIAP expression levels were decreased in CNE-1-LMP1 cells by treatment with embelin, and LMP1-induced resistance to TRAIL was prevented. This finding 
indicated that embelin may replace XIAP-siRNA in preventing LMP1-induced TRAIL resistance in NPC cells.

In order to understand the association between LMP1 and XIAP in various NPC cell lines, the expression levels of XIAP in three NPC cell lines and NP-69 was tested. Notably, the expression levels of XIAP were not high in C666-1 cells, but CNE-2 cells demonstrated the highest expression levels, suggesting that LMP1 was not the only regulatory factor of XIAP expression. Embelin was indicated to enhance TRAIL-induced apoptosis in C666-1 cells, an EBV-positive NPC cell line, and additionally suggested that embelin may sensitize EBV-positive NPC patients to TRAIL treatment. However, a significantly decreased apoptotic response was demonstrated in C666-1 cells that were treated with embelin and TRAIL, compared with CNE-1-LMP1 cells. The difference in these responses may be attributable to the various cell sources and other EBV genes. In Fig. 6E, embelin did not affect TRAIL-induced apoptosis in NP-69 cells, suggesting that embelin failed to enhance TRAIL-induced apoptosis in benign cells. The results of the present study are considered to indicate that combined treatment with embelin fails to augment the toxicity of TRAIL.

In conclusion, the present study has shown that, following the overexpression of LMP1, NPC cells acquired resistance to TRAIL-mediated apoptosis through the upregulation of XIAP. Embelin may exhibit efficacy in combinatorial therapy with TRAIL in an attempt to manage EBV-positive NPC patients.

\section{References}

1. Walczak H and Krammer PH: The CD95 (APO-1/Fas) and the TRAIL (APO-2L) apoptosis systems. Exp Cell Res 256: 58-66, 2000.

2. Pan G, Ni J, Wei YF, Yu G, Gentz R and Dixit VM: An antagonist decoy receptor and a death domain-containing receptor for TRAIL. Science 277: 815-818, 1997.

3. Kaufmann SH and Earnshaw WC: Induction of apoptosis by cancer chemotherapy. Exp Cell Res 256: 42-49, 2000.

4. Luo X, Budihardjo I, Zou H, Slaughter C and Wang X: Bid, a $\mathrm{Bcl}-2$ interacting protein, mediates cytochrome c release from mitochondria in response to activation of cell surface death receptors. Cell 94: 481-490, 1998.

5. Siddiqui IA, Malik A, Adhami VM, Asim M, Hafeez BB Sarfaraz S and Mukhtar H: Green tea polyphenol EGCG sensitizes human prostate carcinoma LNCaP cells to TRAIL-mediated apoptosis and synergistically inhibits biomarkers associated with angiogenesis and metastasis. Oncogene 27: 2055-2063, 2008.

6. Yamaguchi K, Uzzo RG, Pimkina J, Makhov P, Golovine K, Crispen P and Kolenko VM: Methylseleninic acid sensitizes prostate cancer cells to TRAIL-mediated apoptosis. Oncogene 24: 5868-5877, 2005.

7. Tang L, Mao Y, Liu L, Liang S, Chen Y, Sun Y, Liao X, Lin A Liu M, Li L and Ma J: The volume to be irradiated during selective neck irradiation in nasopharyngeal carcinoma: Analysis of the spread patterns in lymph nodes by magnetic resonance imaging. Cancer 115: 680-688, 2009.

8. Isobe K, Ito H, Shigematsu N, Kawada T, Yasuda S, Hara R, Machida N, Takano H, Uchida Y, Uno T, et al: Advanced nasopharyngeal carcinoma treated with chemotherapy and radiotherapy: Distant metastasis and local recurrence. Int J Oncol 12: 1183-1187, 1998.

9. Shao JY, Wang HY, Huang XM, Feng QS, Huang P, Feng BJ, Huang LX, Yu XJ, Li JT, Hu LF, et al: Genome-wide allelotype analysis of sporadic primary nasopharyngeal carcinoma from southern China. Int J Oncol 17: 1267-1275, 2000

10. Teng MS, Brandwein Gensler MS, Teixeira MS, Martignetti JA and Duffey DC: A Study of TRAIL receptors in squamous cell carcinoma of the head and neck. Arch Otolaryngol Head Neck Surg 131: 407-412, 2005.
11. Chen LC, Chung IC, Hsueh C, Tsang NM, Chi LM, Liang Y, Chen CC, Wang LJ and Chang YS: The antiapoptotic protein, FLIP, is regulated by heterogeneous nuclear ribonucleoprotein $\mathrm{K}$ and correlates with poor overall survival of nasopharyngeal carcinoma patients. Cell Death Differ 17: 1463-1473, 2010.

12. Ozören N, Fisher MJ, Kim K, Liu CX, Genin A, Shifman Y, Dicker DT, Spinner NB, Lisitsyn NA and El-Deiry WS: Homozygous deletion of the death receptor DR4 gene in a nasopharyngeal cancer cell line is associated with TRAIL resistance. Int J Oncol 16: 917-925, 2000.

13. Li SS, Tang QL, Wang SH, Wang S and Yang XM: Simultaneously targeting bcl-2 and Akt pathways sensitizes nasopharyngeal carcinoma to tumor necrosis factor-related apoptosis-inducing ligand. Cancer Biother Radiopharm 27: 88-95, 2012.

14. Kijima T, Kinukawa N, Gooding WE and Uno M: Association of Epstein-Barr virus with tumor cell proliferation: Clinical implication in nasopharyngeal carcinoma. Int J Oncol 18: 479-485, 2001.

15. Tang YL, Lu JH, Cao L, Wu MH, Peng SP, Zhou HD, Huang C, Yang YX, Zhou YH, Chen Q, et al: Genetic variations of EBV-LMP1 from nasopharyngeal carcinoma biopsies: Potential loss of $\mathrm{T}$ cell epitopes. Braz J Med Biol Res 41: $110-116,2008$

16. Du CW, Wen BG, Li DR, Peng X, Hong CQ, Chen JY, Lin ZZ, Hong X, Lin YC, Xie LX, et al: Arsenic trioxide reduces the invasive and metastatic properties of nasopharyngeal carcinoma cells in vitro. Braz J Med Biol Res 39: 677-685, 2006.

17. Mei YP, Zhou JM, Wang Y, Huang H, Deng R, Feng GK, Zeng YX and Zhu XF: Silencing of LMP1 induces cell cycle arrest and enhances chemosensitivity through inhibition of AKT signaling pathway in EBV-positive nasopharyngeal carcinoma cells. Cell Cycle 6: 1379-1385, 2007.

18. Dawson CW, Tramountanis G, Eliopoulos AG and Young LS: Epstein-Barr virus latent membrane protein 1 (LMP1) activates the phosphatidylinositol 3-kinase/Akt pathway to promote cell survival and induce actin filament remodeling. J Biol Chem 278: 3694-7304, 2003

19. Friboulet L, Pioche-Durieu C, Rodriguez S, Valent A, Souquère S, Ripoche H, Khabir A, Tsao SW, Bosq J, Lo KW and Busson P: Recurrent overexpression of c-IAP2 in EBV-associated nasopharyngeal carcinomas: Critical role in resistance to Toll-like receptor 3-mediated apoptosis. Neoplasia 10: 1183-1194, 2008.

20. Hornle M, Peters N, Thayaparasingham B, Vörsmann H, Kashkar H and Kulms D: Caspase-3 cleaves XIAP in a positive feedback loop to sensitize melanoma cells to TRAIL-induced apoptosis. Oncogene 30: 575-587, 2011.

21. Snow AL, Vaysberg M, Krams SM and Martinez OM: EBV B lymphoma cell lines from patients with post-transplant lymphoproliferative disease are resistant to TRAIL-induced apoptosis. Am J Transplant 6: 976-985, 2006.

22. Hitt MM, Allday MJ, Hara T, Karran L, Jones MD, Busson P, Tursz T, Ernberg I and Griffin BE: EBV gene expression in an nasopharyngeal carcinoma-related tumor. EMBO J 8: 2639-2651, 1989.

23. Wang D, Liebowitz D and Kieff E: An EBV membrane protein expressed in immortalized lymphocytes transforms established rodent cells. Cell 43: 831-840, 1985.

24. Lin SY, Tsang NM, Kao SC, Hsieh YL, Chen YP, Tsai CS, Kuo TT, Hao SP, Chen IH and Hong JH: Presence of Epstein-Barr virus latent membrane protein 1 gene in the nasopharyngeal swabs from patients with nasopharyngeal carcinoma. Head Neck 23: 194-200, 2001

25. Zhang X, Sanmun D, Hu L, Fadeel B and Ernberg I: Epstein-Barr virus-encoded LMP1 promotes cisplatin-induced caspase activation through JNK and NF-kappaB signaling pathways. Biochem Biophys Res Commun 360: 263-268, 2007.

26. Wei MC, Zong WX, Cheng EH, Lindsten T, Panoutsakopoulou V, Ross AJ, Roth KA, MacGregor GR, Thompson CB and Korsmeyer SJ: Proapoptotic BAX and BAK: A requisite gateway to mitochondrial dysfunction and death. Science 292: 727-730, 2001.

27. Pratt ZL, Zhang J and Sugden B: The latent membrane protein 1 (LMP1) oncogene of Epstein-Barr virus can simultaneously induce and inhibit apoptosis in B cells. J Virol 86: 4380-4393, 2012.

28. Lee H, Seo SY, Tiwari I and Jang KL: Epstein-Barr Virus latent membrane protein 1 overcomes all-trans retinoic acid-induced apoptosis by inhibiting retinoic acid receptor- $\beta_{2}$ expression. Biochem Biophys Res Commun 423: 313-318, 2012.

29. Thornberry NA and Lazebnik Y: Caspases: Enemies within. Science 281: 1312-1316, 1998. 
30. Vier J, Gerhard M, Wagner H and Häcker G: Enhancement of death-receptor induced caspase-8-activation in the death-inducing signalling complex by uncoupling of oxidative phosphorylation. Mol Immunol 40: 661-670, 2004.

31. Li SS, Yang S, Wang S, Yang XM, Tang QL and Wang SH: Latent membrane protein 1 mediates the resistance of nasopharyngeal carcinoma cells to TRAIL-induced apoptosis by activation of the PI3K/Akt signaling pathway. Oncol Rep 26: 1573-1579, 2011.

32. Le Clorennec C, Ouk TS, Youlyouz-Marfak I, Panteix S, Martin CC, Rastelli J, Adriaenssens E, Zimber-Strobl U, Coll J, Feuillard $\mathbf{J}$ and Jayat-Vignoles C: Molecular basis of cytotoxicity of Epstein-Barr virus (EBV) latent membrane protein 1 (LMP1) in EBV latency III B cells: LMP1 induces type II ligand-independent autoactivation of CD95/Fas with caspase 8-mediated apoptosis. J Virol 82: 6721-6733, 2008.

33. Chen Y and Chen XY: Effect of Epstein-Barr virus latent membrane protein 1 (LMP1) on apoptosis of nasopharyngeal carcinoma cell line CNE-1. Ai Zheng 21: 498-503, 2002 (In Chinese).

34. Wei Y, Fan T and Yu M: Inhibitor of apoptosis proteins and apoptosis. Acta Biochim Biophys Sin (Shanghai) 40: 278-288, 2008.

35. Eckelman BP, Salvesen GS and Scott FL: Human inhibitor of apoptosis proteins: Why XIAP is the black sheep of the family. EMBO Rep 7: 988-994, 2006.

36. Mizutani Y, Nakanishi H, Li YN, Matsubara H, Yamamoto K, Sato N, Shiraishi T, Nakamura T, Mikami K, Okihara K, et al: Overexpression of XIAP expression in renal cell carcinoma predicts a worse prognosis. Int J Oncol 30: 919-925, 2007.

37. Checinska A, Hoogeland BS, Rodriguez JA, Giaccone G and Kruyt FA: Role of XIAP in inhibiting cisplatin-induced caspase activation in non-small cell lung cancer cells: A small molecule Smac mimic sensitizes for chemotherapy-induced apoptosis by enhancing caspase-3 activation. Exp Cell Res 313: 1215-1224, 2007.
38. Ndozangue-Touriguine O, Sebbagh M, Mérino D, Micheau O, Bertoglio J and Bréard J: A mitochondrial block and expression of XIAP lead to resistance to TRAIL-induced apoptosis during progression to metastasis of a colon carcinoma. Oncogene 27: 6012-6022, 2008.

39. Deveraux QL, Leo E, Stennicke HR, Welsh K, Salvesen GS and Reed JC: Cleavage of human inhibitor of apoptosis protein XIAP results in fragments with distinct specificities for caspases. EMBO J 18: 5242-5251, 1999.

40. Riedl SJ, Renatus M, Schwarzenbacher R, Zhou Q, Sun C, Fesik SW, Liddington RC and Salvesen GS: Structural basis for the inhibition of caspase-3 by XIAP. Cell 104: 791-800, 2001

41. Ferreira KS, Kreutz C, Macnelly S, Neubert K, Haber A, Bogyo M, Timmer J and Borner C: Caspase-3 feeds back on caspase-8, Bid and XIAP in type I Fas signaling in primary mouse hepatocytes. Apoptosis 17: 503-515, 2012.

42. Feresin GE, Tapia A, Sortino M, Zacchino S, de Arias AR, Inchausti A, Yaluff $G$, Rodriguez J, Theoduloz $C$ and Schmeda-Hirschmann G: Bioactive alkyl phenols and embelin from Oxalis erythrorhiza. J Ethnopharmacol 88: 241-247, 2003.

43. Nikolovska-Coleska Z, Xu L, Hu Z, Tomita Y, Li P, Roller PP, Wang R, Fang X, Guo R, Zhang M, et al: Discovery of embelin as a cell-permeable, small-molecular weight inhibitor of XIAP through structure-based computational screening of a traditional herbal medicine three-dimensional structure database. J Med Chem 47: 2430-2440, 2004.

44. Mori T, Doi R, Kida A, Nagai K, Kami K, Ito D, Toyoda E, Kawaguchi Y and Uemoto S: Effect of the XIAP inhibitor Embelin on TRAIL-induced apoptosis of pancreatic cancer cells. J Surg Res 142: 281-286, 2007. 